\title{
A Proposal to Model Ancient Silk Weaving Techniques and Extracting Information from Digital Imagery - Ongoing Results of the SILKNOW Project
}

\author{
Cristina Portalés $^{1(\bowtie)}(\mathbb{D})$, Javier Sevilla ${ }^{1}\left(\mathbb{D}\right.$, Manolo Pérez $^{1}(\mathbb{D}$, \\ and Arabella León ${ }^{2}$ (D) \\ ${ }^{1}$ Universitat de València, Blasco Ibáñez 13, 46013 València, Spain \\ cristina.portales@uv.es \\ 2 Garín 1820 S.A., Ramon Villarroya, 15, 46113 Moncada, València, Spain
}

\begin{abstract}
Three dimensional (3D) virtual representations of the internal structure of textiles are of interest for a variety of purposes related to fashion, industry, education or other areas. The modeling of ancient weaving techniques is relevant to understand and preserve our heritage, both tangible and intangible. However, ancient techniques cannot be reproduced with standard approaches, which usually are aligned with the characteristics of modern, mechanical looms. The aim of this paper is to propose a mathematical modelling of ancient weaving techniques by means of matrices in order to be easily mapped to a virtual 3D representation. The work focuses on ancient silk textiles, ranging from the 15th to the 19th centuries. We also propose a computer vision-based strategy to extract relevant information from digital imagery, by considering different types of images (textiles, technical drawings and macro images). The work here presented has been carried out in the scope of the SILKNOW project, which has received funding from the European Union's Horizon 2020 research and innovation program under grant agreement No. 769504.
\end{abstract}

Keywords: Silk $\cdot$ Weaving $\cdot$ Modelling $\cdot 3 \mathrm{D} \cdot$ Image processing

\section{Introduction}

Knowing the internal structure of textiles is of relevance for a variety of areas, such as fashion, industry, education or even cultural heritage. Indeed, the modeling of ancient weaving techniques such as brocato, damask or espolin can be of relevance to understand and preserve our heritage, both tangible and intangible.

In the field of research, different authors have dealt with the $3 \mathrm{D}$ representation textiles and/or clothes [1-4], although not all of them deal with the internal structure of textiles at the yarn level. A good example is the work presented in [3], where a representation of knitted cloth at the yarn level is proposed, which treats yarn-yarn contacts as persistent, avoiding expensive contact handling altogether. On the other hand, there also exist a variety of software for the $3 \mathrm{D}$ virtual representation of textiles, such as WiseTex [5, 6] or TexGen [7]. However, many of the ancient silk weaving 
techniques cannot be reproduced with standard software solutions, which are usually aligned with the characteristics of modern, mechanical looms. For example, the spines that weave the handlooms cannot be reproduced on modern looms. This is because the wefts representing pictorial parts in these ancient fabrics are centered in certain places (prepared by the weaver), while the mechanical looms go automatic and pass their plots from side to side of the fabric (Fig. 1). Because of that, a new mathematical representation embedding these techniques is needed, that allows an easy transfer to 3D drawing tools.

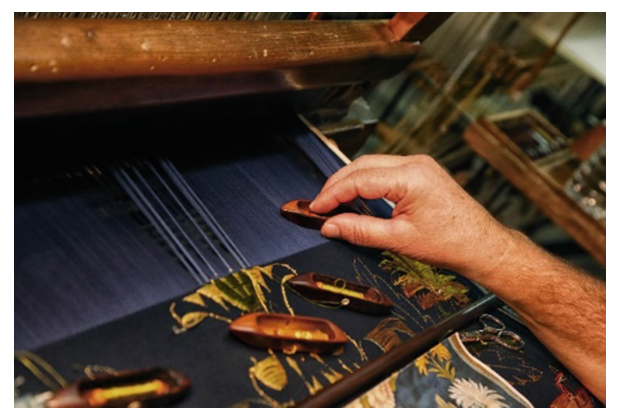

Fig. 1. Making a Valencian espolin with a Jacquard loom. It can be seen how the golden threads do not go from side to side of the fabric, but remain concentrated around the pictorial motifs.

The aim of this paper is to propose a basic mathematical representation of ancient weaving techniques, focusing on ancient silk textiles, ranging from the 15th to the 19th centuries. We also propose a computer vision-based strategy to extract relevant information from digital imagery, by considering different types of images. The work here presented has been carried out in the scope of SILKNOW [8], an ongoing threeyear project (2018-2021) that aims at preserving silk digital heritage. As part of this project, a module called Virtual Loom will be designed and implemented. This module is meant to preserve the fragile ancient art of weaving by directly "cloning" the way it was woven. By means of this tool, high resolution 3D models of the textiles will be produced, based on mathematical models of weaving techniques and the pictorial forms embedded in images. Having information about the weaving technique (to which mathematical models will be defined) and the image itself (mainly, technical drawings and textiles images), virtual models of the fabrics will be automatically produced with a virtual reality motor generation-based software, such as Unity 3D [9], that additionally allows the generation of multi-platform solutions.

\section{Representation of Ancient Weaving Techniques}

\subsection{Single Matrix Representation}

In Fig. 2, the representation of a basic weave is given, following the usual convention. As it can be seen, this example can be fully represented with only one matrix. The given 
matrix indicates the level of the warp, with level 0 indicating the surface of the textile, and the level 1 indicating the level below the textile. As the threads interact one with other, the wefts lay just in the other level: when a warp is in level 0, the weft is in level 1 and the other way round. The example shown in Fig. 2 correspond to a plain weave or tabby. Other basic weaves such as twill and satin, can be also represented in this way.

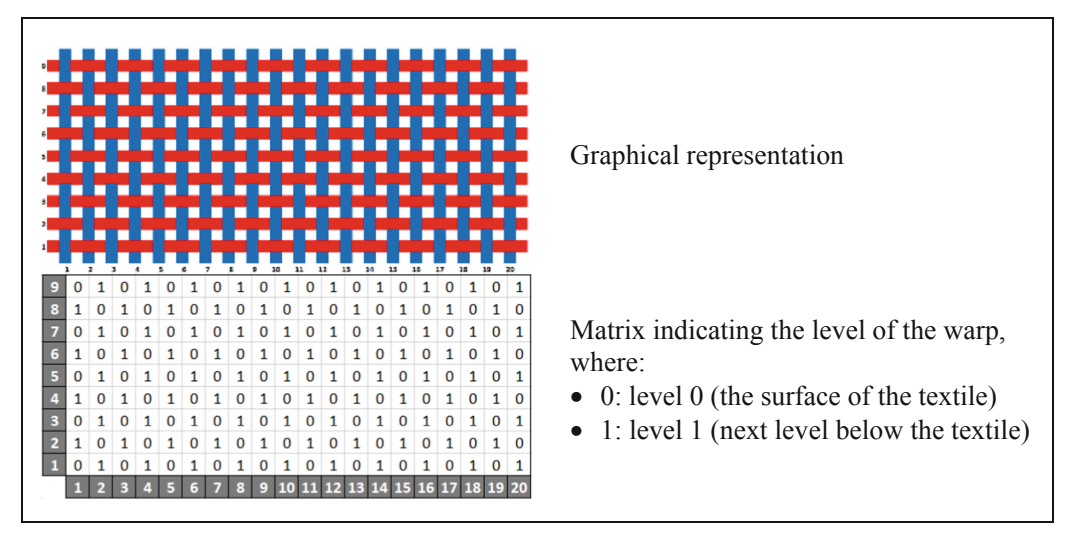

Fig. 2. Representation of a fabric with a basic weave (plain weave or tabby). The warp (vertical yarns) are shown in blue color; the weft (horizontal yarns) are represented in red color. No pictorial parts are considered in this example. (Color figure online)

\subsection{Multiple Matrix Representation}

Matricial forms can also be used to represent complex weavings involving different layers, as suggested by $[10,11]$, where the topological coding of a multilayered weave is based on warp yarns paths. However, for the representation of ancient silk textiles more than one matrix is needed. This is because the yarns representing pictorial parts in these ancient fabrics are centered in certain places (prepared by the weaver), so they do not go from side to side of the fabric. As they are horizontally weaved, they are aligned with the weft yarns, but constitute an additional layer that is not homogeneous alongside with the textile.

Therefore, pictorial parts involving other threads need to be represented with additional matrices, such as the one we propose in Fig. 3. Representing the threads of pictorial parts independently and considering both sides of the textile, allows accounting from possible overlays on the backside. This could be done simple looking where there are "ones" for a same cell in matrices representing threads of different colors. For the 3D modelling, a logical order needs to be established to see in what level representing each thread for each cell. As all the matrices have the same size (rows, columns), the complete representation of a textile can be in form of a hypercube with different dimensions (Di), which will be ordered in the following way: 
- $\mathrm{D}_{0}$ : the matrix embedding the pattern or background

- $\mathrm{D}_{1}$ : the matrix of the pictorial part of yarn 1 , for the front side of the textile

- $\mathrm{D}_{2}$ : the matrix of the pictorial part of yarn 1 , for the backside of the textile

- ...

- $\mathrm{D}_{\mathrm{n}-1}$ : the matrix of the pictorial part of yarn $\mathrm{n} / 2$, for the front side of the textile

- $\mathrm{D}_{\mathrm{n}}$ : the matrix of the pictorial part of yarn $\mathrm{n} / 2$, for the backside of the textile

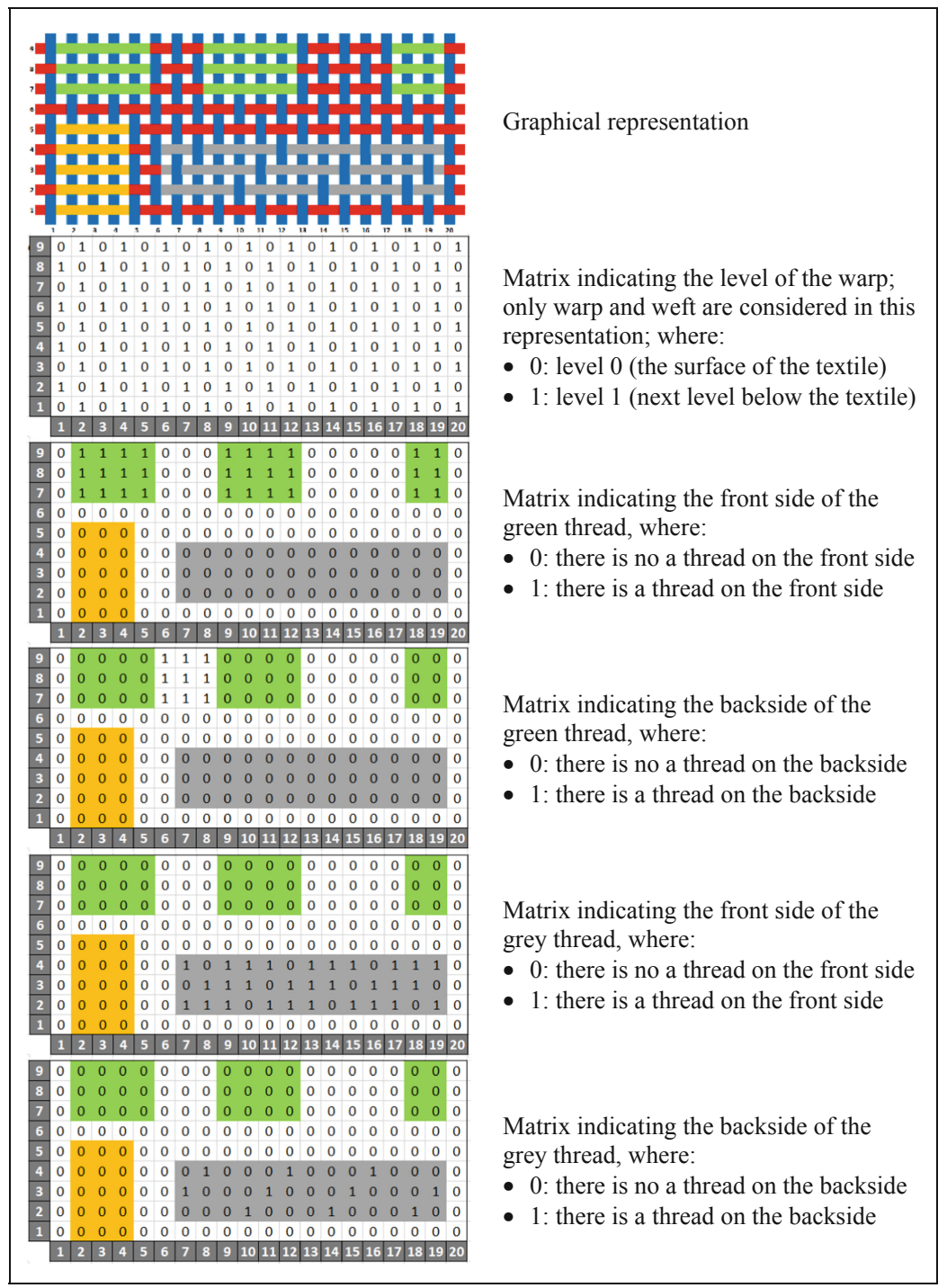

Fig. 3. Representation of a textile with pictorial parts. For the sake of brevity, the two matrices corresponding to the orange yarn have been omitted. 


\section{Extracting Information from Imagery}

\subsection{Types of Images}

Most of the images belonging to the digital collections correspond to photographs of ancient textiles taken with an RGB camera (Fig. 4a). Therefore, this is the main type of information that will be considered for the Virtual Loom. Other types of images can be also used in order to extract more information on the textiles or to complement the RGB images, such as technical drawings (Fig. 4b), macro images (i.e. close-up photography) (Fig. 4c), images in other spectral bands, etc.
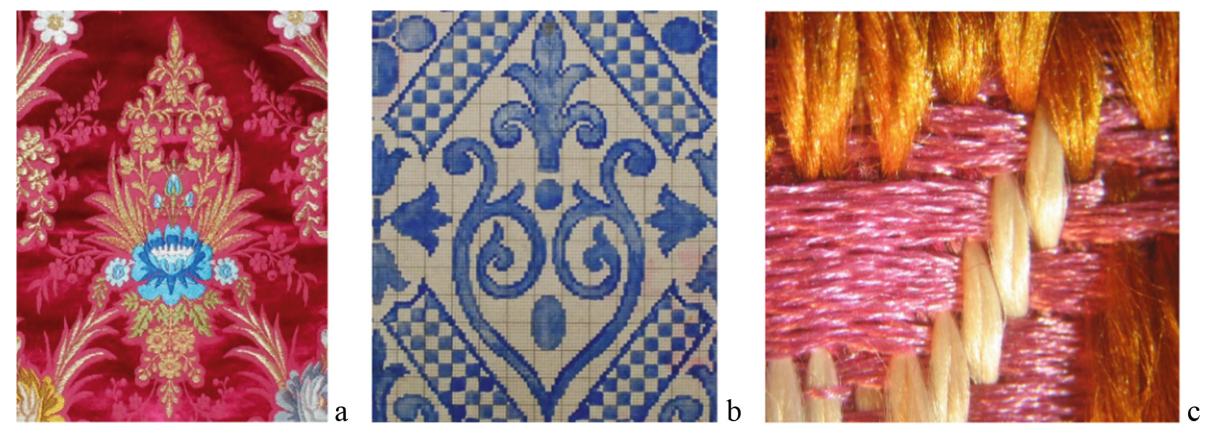

Fig. 4. Examples of images, where: (a) image of a textile (detail); (b) image of a technical drawing (detail); (c) macro image of a textile.

\subsection{Methodology}

Strategy 1: given an image of a textile. The way of proceed in this case is the following (Fig. 5):

- The user will manually select the area interactively.

- If not available from the text, the user will specify the number of different yarns (i.e., the number of colors).

- The image will be posterized to get an image with so many plain colors as number of different yarns.

- The image will be processed in order to extract the background and pictures with different colors. To that end, users will interactively click in one part of the image that contains the background.

- The user will specify, for the background and each of the pictorial part, a weaving technique. For instance, for the background he/she will be able to choose between tabby, twill or satin with different variations.

- If not available from the text, the user will manually specify the number of weft yarns and warp yarns.

- A matrix will be built based on the aforementioned values, following the conventions explained in Sect. 2; rows and columns of the matrix will be build according the number of weft yarns and warp yarns. 
Strategy 2: given an image of a technical drawing of a textile. To extract the number of weft and warp yarns the following procedure is followed (Fig. 6):

- To begin, the original image is converted to greyscale values.

- Then, a local threshold is applied in order to highlight borders. In this way, the original grid is depicted, as schematized in the "thresholded image".

- A morphological operator is used to find crosses in the image. The resulting image will be composed of crosses, although it might happen that some of them are missing if the grid was not fully reproduced in the previous step.

- In order to retrieve pixel coordinates of the centers of the crosses, blobs are computed, which contain such information.

- After ordering the list with the $(x, y)$ pixel values, the number of rows and cols are retrieved. Then, the number of weft and warp yarns are directly computed: number of weft yarns $=$ number of rows +1 ; number of warp yarns $=$ number of cols +1 .

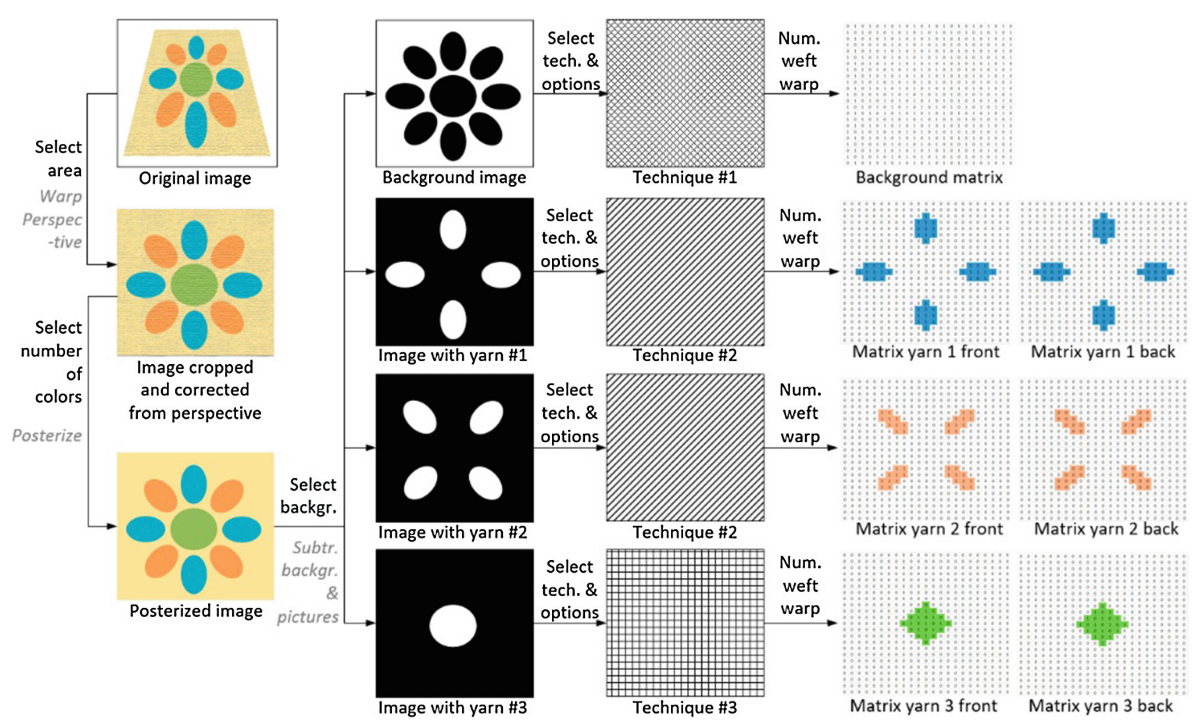

Fig. 5. Methodology used in strategy 1 to derive the matrices that will be used for the Virtual Loom. 


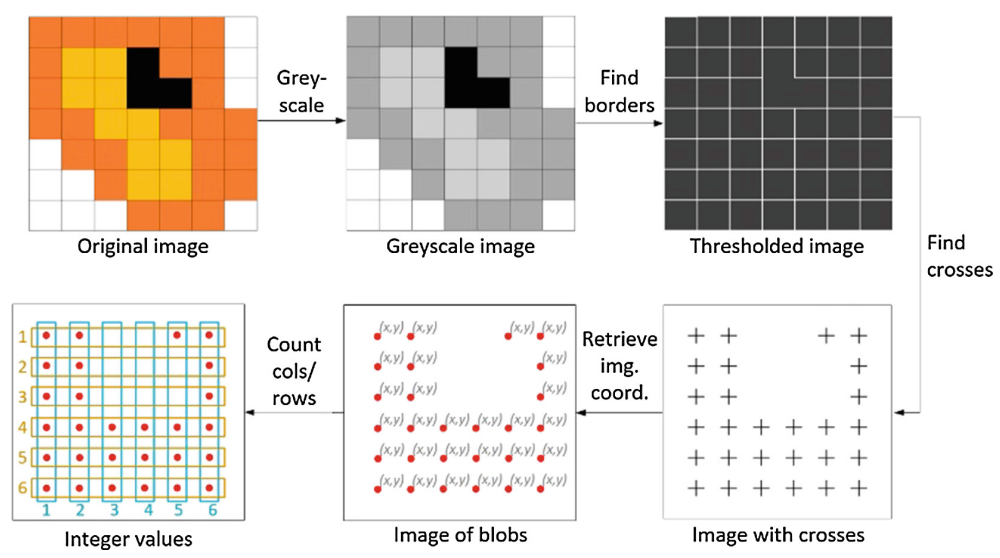

Fig. 6. Methodology used in strategy 2 to derive the number of weft and warp yarns.

\section{Conclusion and Future Work}

Ancient silk textiles are an endangered heritage, not only the textiles themselves, but also their weaving techniques. In order to preserve this legacy, in this paper we show a proposal to represent such techniques with matrices, and to extract information from digital imagery. This information is key to build 3D models of their internal structure, what constitute the next step in our research.

Acknowledgments. The research leading to these results is in the frame of the "SILKNOW. Silk heritage in the Knowledge Society: from punched cards to big data, deep learning and visual/tangible simulations" project, which has received funding from the European Union's Horizon 2020 research and innovation program under grant agreement No. 769504.

\section{References}

1. Etzmuss, O., Keckeisen, M., Strasser, W.: A fast finite element solution for cloth modelling. In: Proceedings of 11th Pacific Conference on Computer Graphics and Applications 200, pp. 244-251 (2003)

2. Jiang, Y., Chen, X.: Geometric and algebraic algorithms for modelling yarn in woven fabrics. J. Text. Inst. 96, 237-245 (2005)

3. Cirio, G., Lopez-Moreno, J., Otaduy, M.A.: Efficient simulation of knitted cloth using persistent contacts. In: Proceedings of the 14th ACM SIGGRAPH/Eurographics Symposium on Computer Animation - SCA 2015, pp. 55-61. ACM Press, Los Angeles (2015)

4. Kaldor, J.M., James, D.L., Marschner, S.: Simulating knitted cloth at the yarn level. In: ACM SIGGRAPH 2008 Papers, pp. 65:1-65:9. ACM, New York (2008)

5. WiseTex Suite. https://www.mtm.kuleuven.be/Onderzoek/Composites/software/wisetex

6. Lomov, S.V.: WiseTex software suite (2005). https://www.mtm.kuleuven.be/Onderzoek/ Composites/software/downloads/wisetex-overview.pdf

7. TexGen. http://texgen.sourceforge.net/index.php/Main_Page 
8. Portalés, C., et al.: Interactive tools for the preservation, dissemination and study of silk heritage - an introduction to the SILKNOW project. Multimodal Technol. Interact. 2, 28 (2018)

9. Unity. https://unity3d.com

10. Lomov, S.V., Huysmans, G., Verpoest, I.: Hierarchy of textile structures and architecture of fabric geometric models. Text. Res. J. 71, 534-543 (2001)

11. Verpoest, I., Lomov, S.V.: Virtual textile composites software WiseTex: integration with micro-mechanical, permeability and structural analysis. Compos. Sci. Technol. 65, 25632574 (2005) 